\title{
Point-of-care ultrasound in management of gallstone ileus - a case report
}

\author{
Magda Iuliana Rotaru ${ }^{1}$, Alexandru Horia Necula ${ }^{2}$, Cosmin Nicolae Caraiani ${ }^{1}$, Alexandru \\ Florin Badea ${ }^{3}$, Marcel Vasile Tantau ${ }^{4}$, Radu Ion Badea ${ }^{1}$
}

${ }^{1}$ Department of Medical Imaging, ${ }^{2} 3$ rd Surgical Department, "Octavian Fodor" Institute of Gastroenterology and Hepatology, ${ }^{3}$ Anatomy and Embryology, Department of Morphological Sciences, ${ }^{4}$ rd Medical Department, "Octavian Fodor" Institute of Gastroenterology and Hepatology, "Iuliu Hatieganu" University of Medicine and Pharmacy, Cluj-Napoca, Romania

\footnotetext{
Abstract

Point-of-care ultrasound has become of utmost importance in the management of critically ill patients, changing everyday clinical practice. With this case report, we highlight the role of abdominal ultrasound in the management of elderly patients with gallstone ileus (a rare cause of intestinal obstructions caused by a cholecysto-duodenal fistula) and with contraindications for contrast-enhanced CT scan.

Keywords: point-of-care ultrasound; cholecysto-duodenal fistula; gallstone ileus; critically ill patients
}

\section{Introduction}

Gallstone ileus is a rare cause of intestinal obstruction due to a cholecysto-duodenal fistula [1]. It typically affects elderly people leading to high morbidity and mortality rates [2]. The contrast enhanced CT (CECT) is the first choice investigation for positive diagnosis [3], but it often cannot be performed due to multiple comorbidities, mainly chronic kidney diseases. Point-of-care ultrasound (US) is an accessible and safe alternative for clinicians to evaluate and to monitor these patients $[4,5]$. We present a case with gallstone ileus; the utility of US in the management of an elderly critically ill patient with gallstone ileus is highlighted.

Received 28.09.2018 Accepted 15.11.2018

Med Ultrason

2019, Vol. 21, No 2, 197-199

Corresponding author: Alexandru Florin Badea, $\mathrm{MD}, \mathrm{PhD}$ Department of Anatomy and Embryology, 3-5 Clinicilor Street, 400006, Cluj-Napoca Phone: 0040755588939

E-mail: alexandru.badea@umfcluj.ro

\section{Case report}

A 86-year-old hypertensive and atherosclerotic woman, with no history of abdominal surgery, was evaluated at the emergency department for nausea, vomiting, abdominal pain and loss of bowel movements lasting for 4-days. She had been previously diagnosed with gallstones but reported no related symptoms.

At admission, she had no fever or jaundice and was hemodynamically stable. The abdomen was painful and mildly distended. She had normal leucocytes, liver and renal function tests but the C-reactive protein (CRP) was high.

CECT of the abdomen was performed for a suspected intestinal obstruction. This was confirmed by dilated jejunal loops. Additionally, the gallbladder had internal gas, calcified content and the wall was thickened. A cholecysto-duodenal fistula was also revealed together with a large hypodense oval image in periumbilical region. These CT findings are suggestive of gallstone ileus (fig $1 \mathrm{a}, \mathrm{b}$ ).

Surgical approach was decided but the patient refused the intervention. Within the further 24 hours, the patient's clinical status worsened: fever, intense abdominal pain with rebound tenderness, together with increasing leukocytes, serum urea, creatinine and CRP. 


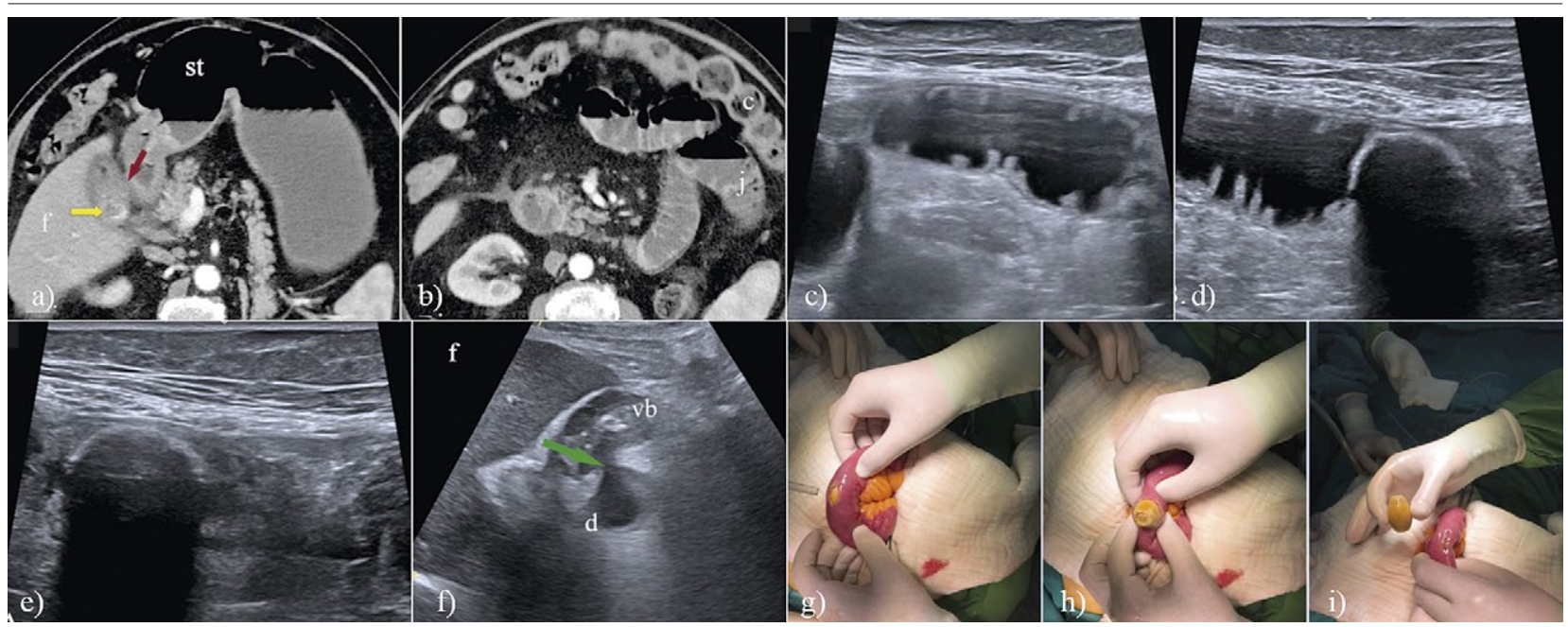

Fig 1. a) and b) Abdominal contrast-enhanced CT (axial view): cholecysto-duodenal fistula (red arrow), cholecystolithiasis (yellow arrow), normal-sized liver [f] (a); small bowel obstruction: fluid-filled distended stomach [st], distended jejunum [j] loops, colon [c] (b); c) Gray-scale abdominal ultrasound: fluid-filled distended intestinal loops with valvulae connivent suggestive for jejunum region; d) and e) Gray-scale abdominal ultrasound showing the fluid-filled jejunal loop behind the obstruction and the ectopic gallstone; f) Gray-scale ultrasound abdominal: fistulous communication (green arrow) between the gall-bladder [vb] and duodenum [d]; $\mathrm{g}), \mathrm{h}$ ) and i) Laparotomy images: the impacted gallstone in proximal jejunum, enterotomy over the site of the impacted gallstone and minimally invasive laparotomy approach.

Abdominal US was performed and demonstrated the Rigler's triad: pneumobilia, intestinal obstruction (fig 1 c), and ectopic gallstone (fig $1 \mathrm{~d}$, e). The site of intestinal obstruction was spotted; a big hyperechoic mass with posterior shadowing, suggestive for a gallstone, was impacted in a jejunal loop at least $20 \mathrm{~cm}$ away from Treitz's angle. Fluid-filled jejunal loop was observed behind the obstruction, together with a little amount of isolated free fluid (fig $1 \mathrm{e}$ ). Further, the US allowed the direct visualization of the cholecysto-duodenal fistula (fig $1 \mathrm{f}$ ).

Afterwards, we obtained the patient's consent for surgery. Therefore, she was referred to department of surgery for emergent intervention. The precise US diagnosis lead to a minimally invasive surgery approach. Laparotomy revealed a large stone, measuring $3 \mathrm{~cm}$ in diameter, impacted in the proximal part of the jejunum and a simple enterolithotomy was performed (fig $1 \mathrm{~g}$-i). Acute inflammatory modifications of the gallbladder were contraindications for cholecystectomy and the fistula itself remained unrepaired. The patient was discharged on the 8 th postoperative with an uneventful recovery.

\section{Discussions}

Cholecystolithiasis is a common health problem. However, gallstone ileus is a rare $(0.3-0.5 \%)$ complication [6]. It affects elderly females [7] and the symptoms are often nonspecific [1]. Delayed diagnosis of this condition leads to high mortality in these patients [8].
Rigler's triad is the specific imaging finding for this disease and can be detected on plain abdominal radiography, abdominal US and CECT [9]. Besides this, identifying the site of intestinal obstruction is important because it guides further therapeutic management: endoscopic versus surgical $[10,11]$.

The CECT evaluation is the first choice investigation for bowel obstruction having a high accuracy (95\%). It also has high diagnostic sensitivity, specificity and accuracy for gallstone ileus $(93 \%, 100 \%$ and $99 \%$, respectively) $[9,12-15]$. However, CT is expensive, involves high doses of radiation and many side effects related to use of iodinated contrast media [16].

Lameris et al [17] suggested that, although CT is the most sensitive imaging investigation for detecting urgent conditions in patients presenting in emergency department, using US first and CT only in those with inconclusive US. This approach leads to a better diagnosis strategy with a significantly lower number of missed urgent conditions compared with $\mathrm{CT}$ only $(6 \%$ versus $11 \%)$.

In our case, both US and CT were able to detect not only the intestinal obstruction, but also the specific location of the ectopic gallstone and direct visualization of the cholecysto-duodenal fistula. However, US was used when CT was contraindicated due to impaired renal function. Moreover, US is safe, accessible, cheap, repeatable, noninvasive, dynamic, in real-time examination. US is highly operator and equipment dependent [18-20] but 
newer techniques are now available to improve the accuracy of the diagnosis.

In this case, we demonstrated that the abdominal US could play an important role in the management of gallstone ileus. With the background of worsened symptoms, point-of-care US evaluation will guided to a better therapeutic decision-making in a critically ill patient with renal failure. The accurate ultrasound diagnosis of the location of intestinal obstruction lead to minimally invasive surgical management. Thereupon, the surgical risks were minimized and the hospitalization period was shortened.

In conclusion, although CECT is considered a gold standard diagnosis tool for gallstone ileus, this case report outlines the advances of US evaluation in critically ill patients with kidney failure.

\section{References}

1. Nuño-Guzmán CM, Marín-Contreras ME, FigueroaSánchez M, Corona JL. Gallstone ileus, clinical presentation, diagnostic and treatment approach. World J Gastrointest Surg 2016;8:65-76.

2. Artioli G, Muri M, Praticò FE, et al. Gallstone ileus: literature review. Acta Biomed 2016;87 Suppl 3:40-44.

3. Chang L, Chang M, Chang HM, Chang AI, Chang F. Clinical and radiological diagnosis of gallstone ileus: a mini review. Emerg Radiol 2018;25:189-196.

4. Croft PE, Noble VE. Point-of-Care Ultrasound. In: O’Donnell J, Nácul F. (eds) Surgical Intensive Care Medicine. Cham: Springer International Publishing; 2016; 787816.

5. Campbell SJ, Bechara R, Islam S. Point-of-Care Ultrasound in the Intensive Care Unit. Clin Chest Med 2018;39:79-97.

6. Ploneda-Valencia CF, Gallo-Morales M, Rinchon C, et al. Gallstone ileus: An overview of the literature. Rev Gastroenterol Mex 2017;82:248-254.
7. Ayantunde AA, Agrawal A. Gallstone Ileus: Diagnosis and Management. World J Surg 2007;31:1292-1297.

8. Reisner RM, Cohen JR. Gallstone ileus: a review of 1001 reported cases. Am Surg 1994;60:441-446.

9. Delabrousse E, Bartholomot B, Sohm O, Wallerand H, Kastler B. Gallstone ileus: CT findings. Eur Radiol 2000;10:938-940.

10. Aguilar-Espinosa F, Maza-Sánchez R, Vargas-Solís F, et al. Fístula colecistoduodenal, complicación infrecuente de litiasis vesicular: nuestra experiencia en su manejo quirúrgico. Rev Gastroenterol Mex 2017;82:287-295.

11. Pavlidis TE, Atmatzidis KS, Papaziogas BT, Papaziogas TB. Management of gallstone ileus. J Hepatobiliary Pancreat Surg 2003;10:299-302.

12. Griffiths S, Glancy DG. Intestinal obstruction. Surgery 2017;35:157-164.

13. Ng YY, Ngu JC, Wong AS. Small bowel obstruction in the virgin abdomen: time to challenge surgical dogma with evidence. ANZ J Surg 2018;88:91-94.

14. Yu CY, Lin CC, Shyu RY, et al. Value of CT in the diagnosis and management of gallstone ileus. World J Gastroenterol 2005;11:2142-2147.

15. Rami Reddy SR, Cappell MS. A Systematic Review of the Clinical Presentation, Diagnosis, and Treatment of Small Bowel Obstruction. Curr Gastroenterol Rep 2017;19:28.

16. Zironi G, Modolon C, Cavazza M. Emergency ultrasound and gallstone ileus. Eur J Emerg Med 2007;14:94-96.

17. Laméris W, van Randen A, van Es HW, et al. Imaging strategies for detection of urgent conditions in patients with acute abdominal pain: diagnostic accuracy study. BMJ 2009;338:b2431.

18. Puylaert JB. Ultrasonography of the acute abdomen. Radiol Clin North Am 2003;41:1227-1242.

19. Pinto A, Pinto F, Faggian A, et al. Sources of error in emergency ultrasonography. Crit Ultrasound J 2013;5 Suppl $1: \mathrm{S} 1$.

20. Chuah PS, Curtis J, Misra N, Hikmat D, Chawla S. Pictorial review: the pearls and pitfalls of the radiological manifestations of gallstone ileus. Abdom Radiol 2017;42:1169-1175. 